ileo-caecal resection were excluded. Demographic data, TI pathology (endoscopic and histopathologic) and indications for colonoscopy were analysed.

Results 8016 colonoscopies were performed with a caecal intubation rate of $90.3 \%$. The endoscopists were of different grades including gastroenterologists, colorectal surgeons and a nurse endoscopist. 206 with ileo-caecal resection were excluded. Mean age was 61 with a female preponderance at $52.6 \%$. The TI $\mathrm{w}$ as intubated in 1845 (23.5\%). Endoscopic TI pathology was identified in 42 patients (2.3\%). Histology was available for 31, of which $23(1.3 \%)$ had confirmed histological abnormalities. Diagnoses on ileoscopy included one adenocarcinoma, one carcinoid tumour, one metastatic malignant melanoma and 20 with terminal ileitis, of which, 6 had histological Crohn's disease. The most common indications in those with TI pathology were diarrhoea (15), abdominal pain (8) and rectal bleeding (8).

Conclusion Although the overall diagnostic yield was low, TI intubation identified significant pathologies requring further action, including three malignancies. Ileoscopy at colonoscopy is a simple manoeuvre, which, apart from quality assurance can identify important pathology. The most common indication in those with confirmed TI pathology was diarrhoea, therefore ileoscopy may have added diagnostic value in this context.

Disclosure of Interest None Declared.

\section{PWE-063 COLONOSCOPY IN THE OVER NINETIES: HOW USEFUL AND SAFE IS IT?}

SK Butt*, A Chitembwe, K Besherdas. Gastroenterology, Chase Farm Hospital, London, UK

10.1136/gutjnl-2014-307263.323

Introduction Colonoscopy is accepted as the gold standard imaging modality of the colon for investigation of colonic symptoms including altered bowel habit, anaemia, rectal bleeding and for the detection of colorectal cancer. The incidence of pathological findings within the colon in particular colorectal cancer increases with age, and the detection of these cancers comprise a major role of colonoscopy in the elderly population. However, colonoscopy in the elderly may be more difficult, as elderly patients are more likely than younger patients to have poor bowel preparations. Furthermore, complications of colonoscopy, in particular cardiovascular, maybe higher in this age group. To date no data exist in colonoscopies performed in those over 90 years of age.

Methods The aim of the study was to assess the diagnostic yield and safety of colonoscopy in the very elderly patients over 90 years of age. A single centre, retrospective analysis of patients over 90 years of age in a district general hospital from north London was performed. The patients were identified using the Unisoft Endoscopy reporting software over a period between June 2006 to March 2013. Data obtained during the study period was scrutinised for indication, sedation administered, quality of bowel prep, findings of procedure, and complications. Results During the study period, 66 colonoscopies were performed out of a total 666 endoscopic procedures in patients over 90 . The indications for colonoscopy included : abnormal CT scan 5/66, altered bowel habit in $17 / 66$, anaemia 24/66, rectal bleeding $15 / 66$, colonic obstruction $3 / 66$, previous polyp/ colon cancer 4/66, abdominal mass 1/66. 18/66 patients had poor bowel prep. Findings included normal in $18 / 66$, colo-rectal cancer in $13 / 66$ patients, colonic polyps in $5 / 66$, ischaemic colitis
$1 / 66$, angiodysplasia 2/66, diverticulosis in $31 / 66$. Sedation administered varied from none in $4 / 66$, to midazolam $5 \mathrm{mg}+$ pethidine $50 \mathrm{mg}$ in $2 / 66$. There were no complications from the colonoscopy in 66 patients within the study.

Conclusion The data from this study demonstrates that colonoscopies can be performed safely in patients over 90 years of age with no increase in procedure related mortality. Despite poor bowel preparation encountered in $28 \%$, the diagnostic yield of pathology in patients over 90 having colonoscopy was very high with an abnormality in 48/66 (72\% in this study). Colonoscopy in the very elderly is very safe and worthwhile and clinicians should not be reluctant in referring those over 90 years of age for colonoscopic examination.

Disclosure of Interest None Declared.

\section{PWE-064 MANAGEMENT OF LARGE COLONIC POLYPS IN THE SEVERN DEANERY HOSPITALS: AN AUDIT OF CURRENT PRACTICE}

${ }^{1}$ SW Dixon*, ${ }^{1} \mathrm{~T}$ Valliani, ${ }^{2} \mathrm{~F}$ Fayyaz, ${ }^{3} \mathrm{D}$ Tate, ${ }^{4} \mathrm{E}$ Arthurs, ${ }^{4} \mathrm{~L}$ Lim, ${ }^{4} \mathrm{~A}$ Dhanda, ${ }^{1} \mathrm{M}$ Lockett. ${ }^{1}$ Gastroenterology, North Bristol NHS Trust, Bristol, UK; ${ }^{2}$ Gastroenterology, Royal United Hospitals, Bath, Bath, UK; ${ }^{3}$ Gastroenterology, Gloucestershire Hospitals NHS Trust, Gloucester, UK; ${ }^{4}$ Gastroenterology, University Hospitals Bristol NHS Trust, Bristol, UK

\subsection{6/gutjnl-2014-307263.324}

Introduction Large $(>2 \mathrm{~cm})$ colonic polyps present a challenge to the colonoscopist. The British Colorectal Cancer Screening Programme (BCSP) recently drafted guidelines to standardise their management. We compared our current practice with the proposed guidelines.

Methods This was a retrospective audit in four South-West hospitals with comparison between screening and non-screening patients. Patients were identified using clinical coding. Case notes were reviewed. Polyps were scored using SMSA system to standardise difficulty of endoscopic resection. Data was compared against 9 auditable outcomes.

Results 104 cases were identified (24 BCSP, 80 symptomatic). There was no significant difference in mean size $(2.9 \mathrm{~cm}$ BCSP, $2.7 \mathrm{~cm}$ symptomatic, $\mathrm{p}=0.14)$ or mean SMSA grading $(2.8$ BCSP, 2.9 symptomatic, $\mathrm{p}=0.46) .6$ polyps were malignant $(1$ BCSP; 5 symptomatic); all had position marked by tattoo. 1 malignant polyp was resected endoscopically in the symptomatic group. Mean time to definitive resection was 34 and 30 days (BCSP and symptomatic respectively). Recurrence of adenoma at EMR site was low at 3 months (0/22 BCSP, 1/37 symptomatic) and 12 months (0/22 BCSP, 2/37 symptomatic). However fewer EMR sites were checked if the index endoscopy was performed by a non-BCS colonoscopist (10/13 vs. 8/24). Complication rates were low: 1 haemorrhage requiring admission (symptomatic group); 0 perforations. 17 benign polyps were referred for surgery ( 3 BCSP, 14 symptomatic; $p=0.49$ ). In both groups the mode SMSA score was 4 . Benign polyps were referred directly for surgery; only those polyps with malignant histology were discussed at a formal mutli-disciplinary meeting. There were no deaths or signficant morbidity associated with surgery for bening polyps.

Conclusion Overall management was comparable to draft guidelines. The frequency of large polyps outside BCSP indicates that non-BCS endoscopists will gain experience with these lesions. Approximately $17 \%$ of benign polyps were referred for surgery, similar to other series. However, none had been discussed with local EMR experts. Consequently a large polyp referral pathway has been established at two centres within the region. 


\section{REFERENCE}

Gupta S, Miskovic D, Bhandari P, et al. SMSA scoring system: a novel scoring system for determining the level of difficulty of a polypectomy. Gastrointestinal Endoscopy 2011;73(4S):AB418-9

Disclosure of Interest None Declared.

\section{PWE-065 COLONOSCOPY PERFORMANCE IN A DISTRICT GENERAL HOSPITAL. HAS THE STANDARDISATION OF TRAINING STANDARDISED PERFORMANCE?}

TM El Menabawey*, J O'Donohue, R Srirajaskanthan. Gastroenterology, University Hospital Lewisham, London, UK

\subsection{6/gutjnl-2014-307263.325}

Introduction Since the introduction of the JAG endoscopy training system (JETS) for trainees in 2003 there have been demonstrable improvements in the key performance indicators (KPIs) of colonoscopy performance. Caecal intubation, polyp detection and polyp retrieval rates are audited KPIs for departments. Terminal ileum (TI) intubation rates are also recorded. The national colonoscopy audit has shown a disparity between medical and surgical performance, but little has been studied to assess if this has improved over time.

Methods We retrospectively audited these KPIs between 2004 and 2012, analysing for variations in performance for all colonoscopists encompassing both trainees and Consultants. We compared the performance of medics and surgeons for each year, the performance in 2004 with 2012 and the overall performance for 9 years using the Chi-squared test.

Results 10055 colonoscopies were performed over 9 years: 8938 by medics and 1117 by surgeons. Completion rates improved significantly from 2004 to 2012 for all colonoscopists $(80.3$ to $92.0 \%, \mathrm{p}<0.001)$. A significant improvement in both specialties' completion rates was seen (medics: 84.1 to $93.0 \%$, surgeons: 74.8 to $88.5 \%, \mathrm{p}<0.001$ ). Over 9 years the overall completion rate for medics was higher $(90.2$ vs. $86.0 \%, \mathrm{p}<$ 0.001). Between 2007 and 2011 there was no significant difference in completion rates. Both specialties' TI intubation rate improved between 2004 and 2012 (medics: 46.3 to 64.1\%, surgeons: 10.41 to $42.0 \%, \mathrm{p}<0.001)$. Overall surgeons were better at polyp detection ( 28.5 vs. $24.8 \%, \mathrm{p}<0.001)$. Surgical performance has not improved since 2004 (29.02 to 23.2\% in 2012), whereas medics improved from 14.4 to $29.7 \%$ ( $\mathrm{p}<$ 0.001 ) to a standard in line with surgical colleagues. Over 9 years there was no significant difference in polyp retrieval rates between specialties (74.9 vs. $76.7 \%$ respectively, $p=0.3)$ and the performance of both improved from 2004 to 2012 (medics: 44.2 to $90.9 \%$, surgeons: 57.6 to $80.2 \%, \mathrm{p}<0.001)$.

Conclusion There has been an overall improvement in colonoscopists' performance in all KPIs between 2004 and 2012. When performance is sub-divided into specialties, one can see that there were significant discrepancies in performance between physicians and surgeons in 2004. With the exception of TI intubation, performance has converged to a similar and higher standard in 2012. This coincides with the introduction of JETS and suggests standardised training may have served to normalise and improve the standard of colonoscopy across both specialties.

\section{REFERENCES}

Gavin DR et al. The national colonoscopy audit: a nationwide assessment of the quality and safety of colonoscopy in the UK. Gut 2012

Kelly NM, et al. Is the 'driving test' a robust quality indicator of colonoscopy performanceaWorld J Gastrointest Endosc 2010

Disclosure of Interest None Declared.

\section{PWE-066 ENDOSCOPIC VERSUS HISTOLOGICAL ASSESSMENT OF COLONIC POLYP SIZE}

WJ Gashau*, C Kong, HY Lee, R Willert. Central Manchester Foundation Trust, Manchester, UK

\subsection{6/gutjnl-2014-307263.326}

Introduction Colonic polyp size is a factor in determining management and prognosis of patients. Polyp diameters greater than $9 \mathrm{~mm}$ require ongoing colonoscopic surveillance. ${ }^{1}$ Accurate endoscopic estimation of polyp size can be affected by depth perception and parallax errors. We compared endoscopic versus histological size assessments to determine if accurate estimation was operator-dependent.

Methods Symptomatic and asymptomatic (bowel screening) patients were identified from hospital databases. Endoscopic and histological polyp diameters were reviewed. Agreement levels between these were analysed by deriving intraclass correlation coefficient (ICC) using SPSS software (Version 20).

Results Sixteen colonoscopists were included: 5 bowel screening, 7 non-bowel screening and 4 trainees. Five hundred and ten polyps $(\mathrm{n}=510)$ were found among 299 patients (186 males, 104 females). Two hundred eighteen polyps were enbloc resected, retrieved whole and analysed. Overall accuracy of polyp size assessment was good (ICC $>0.70$ ) with variability between skill levels (Table 1).

Accuracy was best among polyps $\geq 20 \mathrm{~mm}$ diameter (ICC $0.99, \mathrm{p}<0.001)$; all removed by bowel screening colonoscopists. Polyps between 8 to $12 \mathrm{~mm}$ demonstrated poor correlation (ICC (absolute agreement) 0.535, P = 0.002).

Conclusion While endoscopic estimation of polyp diameter is accurate, variability exists. Estimations are more accurate among bowel screening endoscopists suggesting experience and/or colonoscopy workload contribute to this skill. Poor diameter estimations among polyps $8-12 \mathrm{~mm}$ has implications for polyp surveillance intervals. Standardising diameter using against closed or open biopsy forceps (width 2.2 and $8 \mathrm{~mm}$ respectively) to optimise accuracy should be used.

\section{REFERENCE}

1 Cairns, et al. Guidelines for colorectal cancer screening and surveillance in moderate and high risk groups (update from 2002). Gut 2010;59:666-690

Disclosure of Interest None Declared.

\begin{tabular}{lcc}
$\begin{array}{l}\text { Abstract PWE-066 Table } 1 \\
\text { assessment according to aolonoscopist }\end{array}$ & Reliability analysis of polyp & \\
\hline & Intraclass correlation coefficient & $\mathrm{p}$-value \\
\hline All & 0.95 & $\mathrm{p}<0.001$ \\
Bowel screening colonoscopists & 0.96 & $\mathrm{p}<0.001$ \\
Non-bowel screening colonoscopists & 0.74 & $\mathrm{p}<0.001$ \\
All trainees (consultant-supervised) & 0.86 & $\mathrm{p}<0.001$ \\
\hline
\end{tabular}

\section{PWE-067 DOES ENDOCUFF-VISION IMPROVE ADENOMA DETECTION}

ZP Tsiamoulos*, K Patel, T Elliott, R Misra, S Thomas-Gibson, C Fraser, A Haycock, BP Saunders. Wolfson Unit for Endoscopy, St Mark's Hospital/Academic Institute, London, UK

10.1136/gutjnl-2014-307263.327 